\title{
A musculoskeletal shoulder model based on pseudo-inverse and null-space optimization
}

\author{
Alexandre Terrier ${ }^{\mathrm{a}, *}$, Martin Aeberhard ${ }^{\mathrm{a}}$, Yvan Michellod ${ }^{\mathrm{b}}$, Philippe Mullhaupt ${ }^{\mathrm{b}}$, \\ Denis Gillet ${ }^{\mathrm{b}}$, Alain Farron ${ }^{\mathrm{c}}$, Dominique P. Pioletti ${ }^{\mathrm{a}}$ \\ a Laboratory of Biomechanical Orthopedics, Ecole Polytechnique Fédérale de Lausanne, Switzerland \\ ${ }^{\mathrm{b}}$ Automatic Control Laboratory, Ecole Polytechnique Fédérale de Lausanne, Switzerland

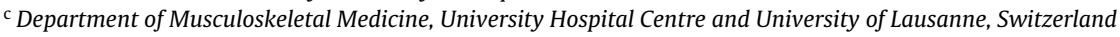

\section{A R T I C L E I N F O}

\section{Article history:}

Received 17 December 2009

Received in revised form 19 May 2010

Accepted 14 July 2010

\section{Keywords:}

Shoulder

Musculoskeletal modeling

Dynamics

\begin{abstract}
A B S T R A C T
The goal of the present work was assess the feasibility of using a pseudo-inverse and null-space optimization approach in the modeling of the shoulder biomechanics. The method was applied to a simplified musculoskeletal shoulder model. The mechanical system consisted in the arm, and the external forces were the arm weight, 6 scapulo-humeral muscles and the reaction at the glenohumeral joint, which was considered as a spherical joint. The muscle wrapping was considered around the humeral head assumed spherical. The dynamical equations were solved in a Lagrangian approach. The mathematical redundancy of the mechanical system was solved in two steps: a pseudo-inverse optimization to minimize the square of the muscle stress and a null-space optimization to restrict the muscle force to physiological limits. Several movements were simulated.

The mathematical and numerical aspects of the constrained redundancy problem were efficiently solved by the proposed method. The prediction of muscle moment arms was consistent with cadaveric measurements and the joint reaction force was consistent with in vivo measurements.

This preliminary work demonstrated that the developed algorithm has a great potential for more complex musculoskeletal modeling of the shoulder joint. In particular it could be further applied to a non-spherical joint model, allowing for the natural translation of the humeral head in the glenoid fossa.

(c) 2010 IPEM. Published by Elsevier Ltd. All rights reserved.
\end{abstract}

\section{Introduction}

From a biomechanical point of view, the shoulder is certainly the most complex human joint. The shoulder is indeed capable of complex kinematics, controlled by a complex muscular system. Even when limited to the 3 rotational degrees of freedom of the glenohumeral joint, shoulder models are mathematically indeterminate, with more muscles (unknowns) than degrees of freedoms (dynamic equations).

To achieve the biomechanical analysis of such a complex system, various simplifications have been proposed. The most straightforward simplification is to limit the analysis to the glenohumeral joint, and to consider only rotational degrees of freedom, assuming that the glenohumeral joint is a spherical (ball and socket) joint. In addition, when the motion is limited to the arm elevation in the

\footnotetext{
* Corresponding author at: Laboratory of Biomechanical Orthopedics, Ecole Polytechnique Fédérale de Lausanne, Station 15, 1015 Lausanne, Switzerland. Tel.: +412169394 98; fax: +41216938660.

E-mail addresses: alexander.terrier@epfl.ch, alexandre.terrier@epfl.ch (A. Terrier).
}

scapular plane, a 2D analysis reduces the degrees of freedom to one elevation angle. The modeling can be further simplified by grouping muscles and neglecting others. This extreme simplification was initially proposed by Inman in his estimate of the glenohumeral reaction force [1]. Poppen and Walker proposed a more complex model by considering 6 glenohumeral muscles [2]. They solved the mechanical indeterminacy of the muscle recruitment by imposing relative muscular activation based on electromyography (EMG) measurements. A similar approach was used in a 3D model of the glenohumeral joint allowing for the natural translation of the humeral head $[3,4]$.

Another way of dealing with the indeterminacy is inverse dynamics coupled with the optimization of an objective function. The Swedish shoulder model considered 23 muscles in static cases, minimizing of the square muscle stress [5]. Muscles were also constrained to be positive (no compression) and limited by a maximal physiological force derived from the Fick law [6]. A maximum glenohumeral force of about $80 \%$ of the body weight (BW) was predicted when the arm was elevated at $60^{\circ}$, with $1 \mathrm{~kg}$ in the hand. Karlsson and Peterson reported some discrepancies with the results of Poppen and Walker and with other EMG measures. Later, the Dutch (Delft) model accounted for the subluxation cri- 


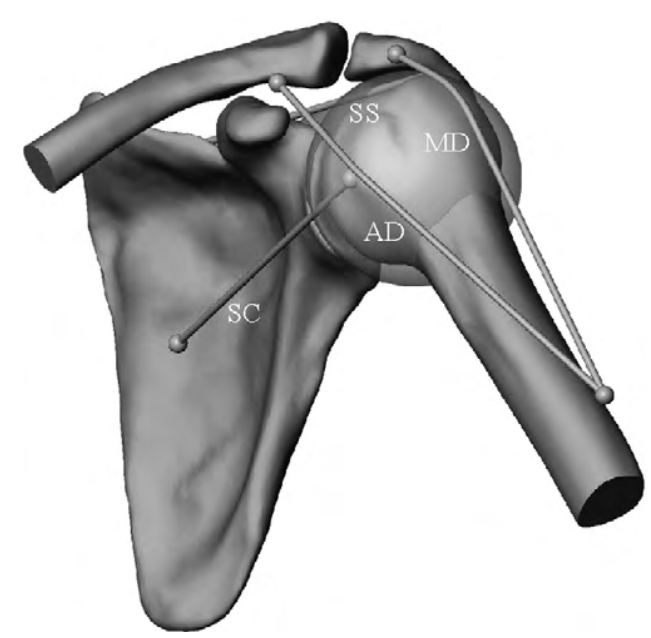

Fig. 1. The system is composed of the humerus, scapula and 6 glenohumeral muscles. The three deltoid parts (MD, AD and PD) were constrained to wrap on an outer sphere (transparent sphere in the figure) fitting the humerus tuberosities. The three rotator cuff muscles (SS, SC, IS) were constrained to wrap on two other inner spheres (not represented in the figure) fitting the articular surface of the humerus (see Table 1).

teria, muscle wrapping, ligaments and dynamics effects [7]. This model predicted a maximal force of approximately $50 \%$ of the BW, at $90^{\circ}$ of arm elevation. More recently, the Newcastle model was used to evaluate the shoulder biomechanics in several activities of daily living and provided similar results as the Dutch model [8]. Another similar model predicted glenohumeral reaction force of about $70 \%$ of the BW during quasi-static abduction in the scapular plane [9]. Other optimization models have been developed for posture control or ergonomics analyses [10-12]. More recently, neuro-muscular aspects have also been investigated [13,14]. Several shoulder models are thus available to estimate muscle and joint forces, but there is some discrepancy in their prediction of joint reaction force. Besides, muscle moment arms, which are crucial, are not always given. In addition, because of the relative complexity of these models, which is usually not fully described, it is difficult to compare them and evaluate the reasons for these differences.

Therefore, the goal of this study was to assess a well-known method used in robotics, based on the pseudo-inverse and nullspace optimization for solving the dynamical shoulder system. In a first step, we thus used a simplified musculoskeletal model to evaluate the method. In a future next step, this method will be adapted to an existing model to predict humeral head translation $[3,4]$.

\section{Method}

\subsection{The mechanical system}

The mechanical system (Fig. 1) was composed of the humerus, the scapula and 6 glenohumeral muscles: the middle deltoid (MD), the anterior deltoid (AD), the posterior deltoid (PD), the supraspinatus (SS), the subscapularis (SC), and the infraspinatus combined with the teres minor (IS). The humerus had 3 rotational degrees of freedom. The scapula motion was constrained to the humerus motion according to a scapulo-humeral rhythm of $2: 1$. The origin, insertion and physiological cross-section area (PCSA) of muscles are given in Table 1 [4]. The wrapping of the muscles around the bone was considered and was calculated using the method of shortest path around the surface $[7,15,16]$. We used 3 spheres centered on the joint center for the deltoid and rotator cuff muscles. The sphere radii (Table 1) were chosen to best fit the humeral head, and
Table 1

PCSA, origins and insertions of the muscles (in the initial position), and radii of the wrapping spheres.

\begin{tabular}{|c|c|c|c|c|c|c|}
\hline & \multicolumn{6}{|c|}{ Muscle } \\
\hline & MD & $\mathrm{AD}$ & PD & SS & $\mathrm{SC}$ & IS \\
\hline $\operatorname{PCSA}\left(\mathrm{cm}^{2}\right)$ & 10 & 10 & 10 & 5 & 15 & 15 \\
\hline$X I(\mathrm{~mm})$ & 20 & 20 & 20 & 26 & 5 & 5 \\
\hline$Y I(\mathrm{~mm})$ & 0 & 0 & 0 & 0 & 21 & -21 \\
\hline$Z I(\mathrm{~mm})$ & -90 & -90 & -90 & 7 & 5 & 8 \\
\hline$X O(\mathrm{~mm})$ & 2 & -15 & -55 & -81 & -99 & -97 \\
\hline$Y O(\mathrm{~mm})$ & 0 & 32 & -28 & 0 & 2 & -15 \\
\hline $\mathrm{ZO}(\mathrm{mm})$ & 33 & 29 & 23 & 25 & -23 & -22 \\
\hline$R(\mathrm{~mm})$ & 30 & 30 & 30 & 26 & 22 & 22 \\
\hline
\end{tabular}

also to best fit cadaveric measurements of muscle moment arms [17-19]. The other parameters of the model were the arm weight $w=37.5 \mathrm{~N}$, the distance of the arm weight center from the joint center $d=0.32 \mathrm{~m} \mathrm{[4]}$, the longitudinal and transverse mass moment of inertia of the arm $I_{l}=0.024 \mathrm{~kg} / \mathrm{m}^{2}$ and $I_{t}=0.003 \mathrm{~kg} / \mathrm{m}^{2}[20]$.

The fixed Oxyz and moving coordinate system $O X Y Z$ were centered on the joint rotation. The arm motion was described by the YXY Euler sequence [21], where $\phi$ defines the abduction plane orientation, $\theta$ the abduction angle, and $\psi$ is the axial rotation.

\subsection{The dynamics equations}

The mechanical system was described in generalized coordinates $\vec{q}=(\phi, \theta, \psi)^{T}$ and generalized forces $\vec{Q}=\left(Q_{\phi}, Q_{\theta}, Q_{\psi}\right)^{T}$. The generalized coordinates were the three rotation angles and the generalized forces were the total moment of force of the muscles projected on the $O X Y Z$ coordinate system. In a Lagrangian formulation

$\frac{d}{d t}\left(\frac{\partial L}{\partial \dot{q}_{i}}\right)-\frac{\partial L}{\partial q_{i}}=Q_{i}, \quad i=\phi, \theta, \psi$

the kinetic energy $T$ and potential energy $V$ of the Lagrangian $L=T-V$ were

$$
\begin{aligned}
T= & \frac{1}{2}\left\{\left(I_{t}+m l^{2}\right)[\dot{\phi} \sin (\theta) \sin (\psi)+\dot{\theta} \cos (\psi)]^{2}+I_{l}[\dot{\phi} \cos (\theta)+\dot{\psi}]^{2}\right. \\
& \left.+\left(I_{t}+m l^{2}\right)[\dot{\theta} \sin (\psi)-\dot{\phi} \sin (\theta) \cos (\psi)]^{2}\right\}
\end{aligned}
$$

and

$V=m g l[1-\cos (\theta)]$

For a known motion, Eq. (1) provided a unique solution of the generalized force $\vec{Q}$.

\subsection{Muscle moment arm matrix}

The total moment of force of the 6 muscles was written as the product of a moment arm matrix $B$ and a force vector $\vec{F}$ [22]:

$\vec{M}=\sum_{i=1}^{6} \vec{r}_{i} \times \vec{F}_{i}=\sum_{i=1}^{6} \vec{r}_{i} \times F_{i} \vec{d}_{i}=\sum_{i=1}^{6} \vec{r}_{i} \times \vec{d}_{i} F_{i}=B \vec{F}$

With this choice, $B$ was a $3 \times 6$ matrix containing the muscle moment arms $\vec{r}_{i}$ in the 3 direction, and $\vec{F}$ was a $6 \times 1$ vector containing the muscle force amplitudes. Eq. (4) was then projected in the generalized force space

$\vec{Q} \equiv P \vec{M}=P B \vec{F}=W \vec{F}$

where

$P=\left[\begin{array}{ccc}\sin (\theta) \sin (\psi) & \cos (\theta) & -\sin (\theta) \cos (\psi) \\ \cos (\psi) & 0 & \sin (\psi) \\ 0 & 1 & 0\end{array}\right]$ 
is a $3 \times 3$ projection matrix and $W=P B$ a $3 \times 6$ matrix. $W$ is an immersion map from a 6 -dimension space to a 3-dimension space. The map $W$ contains the muscle moment arms and provides a relationship between the force amplitude of the muscles and the generalized force.

\subsection{System indeterminacy and null-space optimization}

The mechanical system had 6 unknown muscle force amplitudes for the 3 dynamic equations (1). This indeterminacy appears in the moment arms matrix $W$, which is singular and has no inverse to provide a unique set of muscle force amplitude $\vec{F}$ from Eq. (5). The muscle forces were also constraint by two physiological limitations: (1) muscles can only provide a contraction force and (2) this force is limited by a maximum value. The maximal force of each muscle $F_{i}^{\max }$ was estimated as proportional to the muscle PCSA according to the classical Fick law $F_{i}^{\max }=k \cdot \mathrm{PCSA}_{i}$, with $k=40 \mathrm{~N} / \mathrm{cm}^{2}[23]$.

This constrained indeterminate problem was solved in two steps. First, a (linear) optimization was performed to minimize the sum of the squared muscle stress. This was done with Lagrange multipliers $\lambda$ and corresponded to a pseudo-inversion of the matrix $W$. In the second step, the physiological constraint was achieved by quadratic programming in the null space of $W$.

For the first step, the cost function for the Lagrange multipliers was

$G_{1}(\vec{F}, \vec{\lambda})=\vec{F}^{T} E \vec{F}-\vec{\lambda}(W \vec{F}-\vec{Q})$

where $E$ is a $6 \times 6$ diagonal matrix with each diagonal element being the inverse of the square PCSA of the corresponding muscle. The Lagrange optimization gives

$\vec{F}=E^{-1} W^{T}\left(W E^{-1} W^{T}\right)^{-1} \vec{Q}=W^{+} \vec{Q}$

which defines the pseudo-inverse

$W^{+}=E^{-1} W^{T}\left(W E^{-1} W^{T}\right)^{-1}$

of $W$. The pseudo-inverse $W^{+}$provided the muscle forces with minimum square stress from the generalized force $\vec{Q}$

$\vec{F}=W^{+} \vec{Q}$

During the second step, the muscle forces $\vec{F}$ were then constrained to stay within the physiological limits

$0 \leq F_{i} \leq F_{i}^{\max }$

by using quadratic programming. It was defined by the following constraint minimization (finding $\vec{\mu}$ that minimize $G_{2}(\vec{\mu})$ )

$\left\{\begin{array}{l}G_{2}(\vec{\mu})=\frac{1}{2} \vec{\mu}^{T} H \vec{\mu}+\vec{h}^{T} \vec{\mu} \\ A \vec{\mu} \leq \vec{b}\end{array}\right.$

where

$H=\frac{1}{2} N^{T} E N$

$\vec{h}=\vec{F}^{T} E N$

$A=\left[\begin{array}{c}N \\ -N\end{array}\right]$

$\vec{b}=\left(F_{1}^{\max }-F_{1}, \ldots, F_{6}^{\max }-F_{6}, F_{1}-F_{1}^{\min }, \ldots, F_{6}-F_{6}^{\min }\right)^{T}$

and with $N=\operatorname{Null}(W)$ is the null space of the matrix $W . N$ is a $6 \times 3$ matrix formed with all vectors $\vec{x}$ for which $W \vec{x}=0$. Finally, the muscle force vector was

$\vec{F}=W^{+} \vec{Q}+N \vec{\mu}$
In summary, the dynamic equation (1) provided the generalized force $\vec{Q}$, which was then transformed into the muscle force $\vec{F}$, using the pseudo-inverse $W^{+}$. Through both the use of the pseudo-inverse and the null-space optimization, the muscle forces $\vec{F}$ providing a feasible dynamical equilibrium of the mechanical system, minimized the sum of the square muscle stress, and were within the physiological limits. The joint reaction force $\vec{R}$ was then easily deduced from the force equilibrium equation

$m \vec{a}=\sum_{i=1}^{6} \vec{F}_{i}+m \vec{g}+\vec{R}$

where $\vec{a}$ is the acceleration of the center of mass of the arm.

\subsection{Kinematics}

Four simple elevation movements in the scapular plane were simulated (Fig. 2). The first one was a slow (quasi-static) abduction defined by

$\left\{\begin{array}{l}\phi(t)=0 \\ \theta(t)=\pi / 2(\cos (2 \pi t / T)-1) \\ \psi(t)=0\end{array}\right.$

with the period $T=20 \mathrm{~s}$. This movement corresponds to a periodic abduction-adduction, from zero to $180^{\circ}$ of abduction, with a null angular elevation velocity at zero and $180^{\circ}$ of abduction. Although we chose an amplitude of $180^{\circ}$ for sake of simplicity in our mathematical equation, this movement was actually only performed from $0^{\circ}$ to $150^{\circ}$, and was thus performed in $7.3 \mathrm{~s}$. For this movement, we also compared the model prediction with in vivo data of an instrumented prosthesis [24].

The second movement was the same as above, with a conjunct external rotation, according to the following expression

$\left\{\begin{array}{l}\phi(t)=0 \\ \theta(t)=\pi / 2(\cos (2 \pi t / T)-1) \\ \psi(t)=\pi / 8(\cos (2 \pi t / T)-1)\end{array}\right.$

The third movement was the same as the first one, performed ten times faster $(T=2 \mathrm{~s})$. In this case $150^{\circ}$ of abduction was achieved in $0.7 \mathrm{~s}$.

The last movement was also a simple abduction with $\phi(t)=0$ and $\psi(t)=0$, but $\theta(t)$ was obtained from in vivo recorded data [25].

\subsection{Implementation}

The Lagrange equations were solved numerically by the ode 45 solver of MatLab (www.mathworks.com). The moment arm matrix $W$ was obtained algebraically from geometrical parameters of the model, while its pseudo-inverse $W^{+}$and null space matrix $N$ were calculated numerically at each time increment. The quadratic optimization in the null space was performed with the quadprog solver of the optimization toolbox of MatLab.

\subsection{Model output}

For the 4 movements tested, we evaluated the following quantities: moment arms of the muscles in the 3 directions, force amplitude of the muscles, and reaction force at the glenohumeral joint. The muscle moment arms were obtained from the moment arm matrix and projected onto the local coordinate system. The first component corresponded to the orientation of the plane of elevation, the second one to the arm elevation and the third one to the arm axial rotation. The muscle force amplitudes were obtained directly from the muscle force vector (Eq. (17)). Finally, the reaction force at the glenohumeral joint was obtained from the dynamical equilibrium equation (Eq. (18)). 

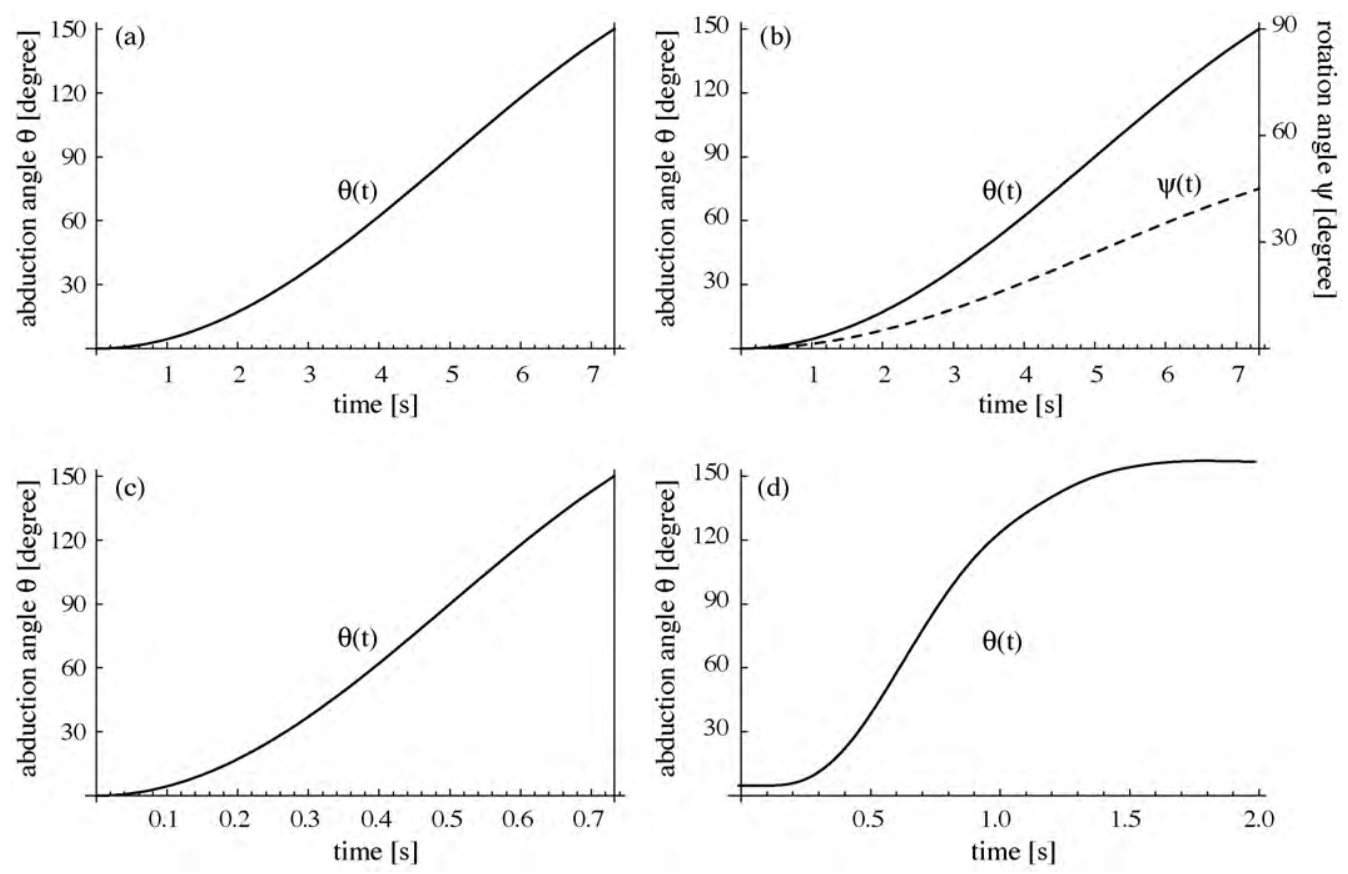

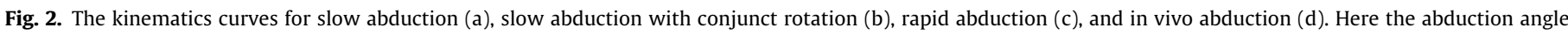

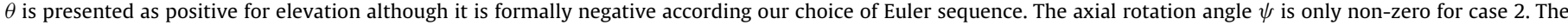
orientation of the plane of elevation $\phi$ is always constant and set to the scapular plane.

\section{Results}

\subsection{Muscle moment arms}

The three components of the muscle moment arms are only presented for the simple abduction case (Fig. 3). The first component corresponds to rotation around the first Euler axis $(Y)$, which is the orientation of the elevation plane. The second one corresponds to the second Euler axis $(X)$, which is elevation. The third one corresponds to the third Euler axis $\left(Y^{\prime}\right)$, which is arm axial rotation. During arm elevation in the scapular plane, the moment arms of the muscles were in overall rather symmetrical. In the $Y$ (elevation plane) and $Y^{\prime}$ axis (arm rotation), the AD and the PD, as the SC and the IS, were nearly antagonists to each other, while the MD and the SS were zero. In the $Y^{\prime}$ axis, the moment arm of the SC and IS were important at low abduction angles, but decreased as abduction increased. In the X direction (arm elevation), the moment arm of the MD was the largest elevating moment arm, followed by the SS. The SC and IS moment arms were much lower. The AD had a depressing moment arm below $30^{\circ}$ of abduction, but was constantly increasing. The PD had a depressing moment arm up to $110^{\circ}$ of abduction.

\subsection{Slow abduction}

For the simple quasi-static abduction, the MD was the dominant muscle. The $\mathrm{AD}$ and $\mathrm{PD}$, were almost inactive below $90^{\circ}$ of abduction, while the SS, SC and IS, followed the same sinusoidal shape. The reaction force versus abduction angle followed approximately the sinusoidal shape of the moment of force of the arm weight (Fig. 4a). The maximal value corresponded to $87 \%$ of the body weight (BW).

The comparison with the in vivo measurement of the reaction force [24] was evaluated at $45^{\circ}$ of abduction. For this angle, the $\phi$

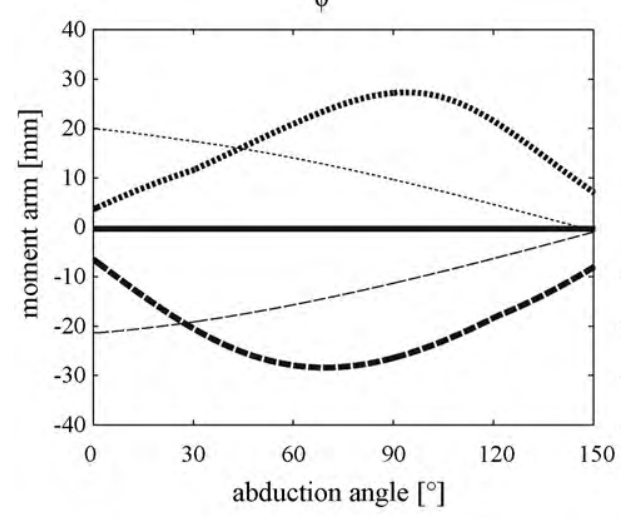

$\theta$

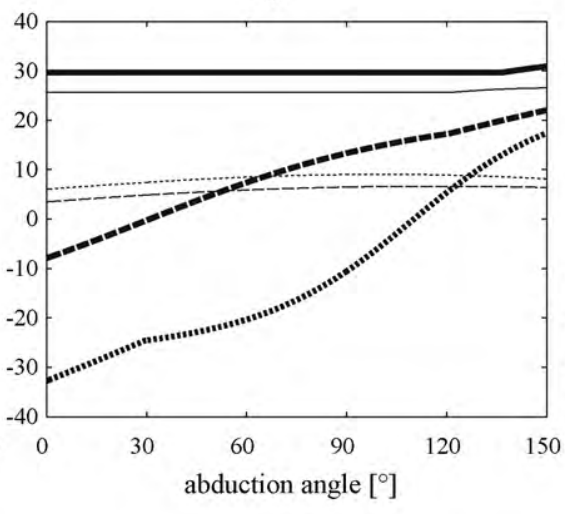

$\psi$

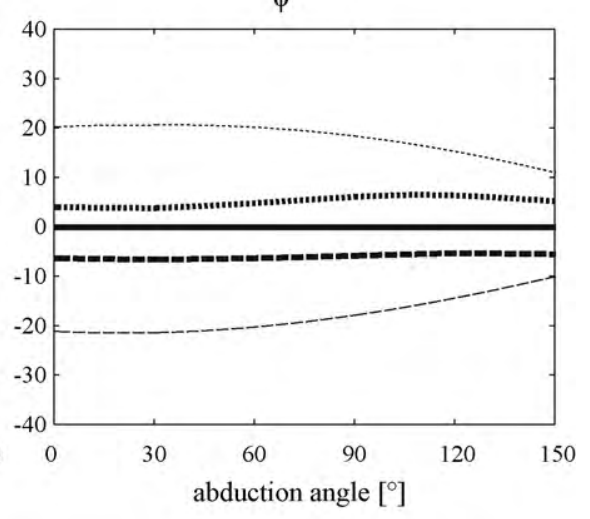

$\mathrm{AD}$ PD SS SC .. IS

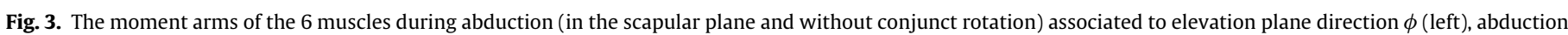
$\theta$ (middle), and axial rotation $\psi$ (right). 

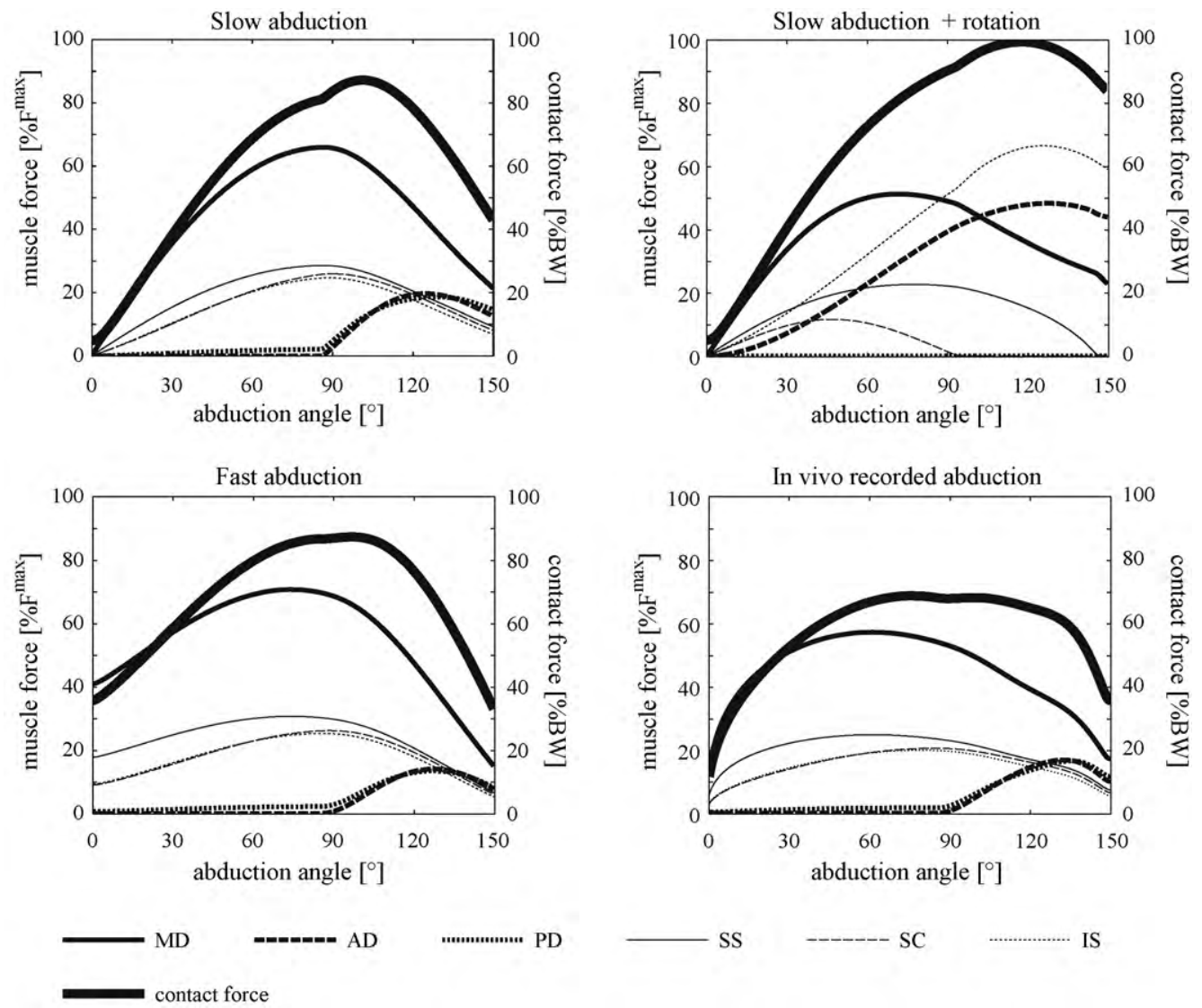

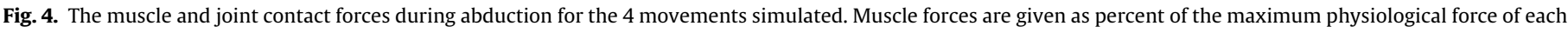
muscle. Joint contact force is given as a percent of the body weight.

predicted reaction force was 95\% BW with $2 \mathrm{~kg}$ in the hand and 52\% BW without any load in the hand, versus $90 \%$ and $50 \%$ respectively in the in vivo study.

\subsection{Slow abduction with conjunct rotation}

When conjunct external rotation was added to slow abduction, the IS was much more active (Fig. 4b). The activation of the IS was associated with the activation of the AD. The SC was less active and even inactive after $90^{\circ}$ of elevation. The PD was completely inactive. The co-activation of the IS and AD were antagonist to each other and increased the joint reaction force up to $99 \% \mathrm{BW}$.

\subsection{Fast abduction}

Performing arm elevation ten times faster than the slow abduction movement (Section 3.2) did not changed the maximum reaction force, which remained $87 \%$ of BW (Fig. 4c vs. Fig. 4a). There was however and increase of the muscle and joint forces at low abduction angles.

\subsection{In vivo recorded abduction}

The duration of the in vivo recorded movement was approximately the same as the above case, but the velocity and acceleration were zero at zero abduction (Fig. 2c). In this case, the maximal reaction force reduced to $73 \%$ of the BW, and it was much lower at the beginning of the movement.

\section{Discussion}

The goal of this paper was to assess the feasibility of a pseudoinverse and null-space optimization to solve the constrained indeterminacy problem associate to the muscle recruitment of the shoulder. The algorithm was tested on simple musculoskeletal model $[4,26]$ and shown to be efficient in predicting a joint reaction force that was consistent with in vivo measurements. This work was a first step, before implementing this algorithm in an existing musculoskeletal shoulder model allowing natural humerus translations to get the joint contact pattern [4]. This musculoskeletal shoulder model is indeed currently using available EMG data and is thus limited to simple movements. The algorithm developed here will provide the muscle activation pattern for more complex shoulder movements.

The predicted moment arms were within the range of cadaveric $[17,27-30]$ and in vivo measurements $[2,31,32]$. For the quasistatic movement, the predicted muscular and joint forces were also within the range of other numerical models. When external rotation was performed during arm elevation, the algorithm predicted the activation of the rotation cuff muscle IS, which is coherent with anatomical reality. The co-activation of the $\mathrm{AD}$ is also reasonable to constrain the elevation of the arm in the scapular plane during conjunct external rotation. Doing the elevation movement in a more natural way, by doing it more rapidly than the quasi-static case, mainly changed the reaction force when the arm is along the body $\left(0^{\circ}\right.$ of elevation $)$. This effect appeared because of the simulated movement was a cyclic movement. Since acceleration, and thus inertial force, is maximal at this position, it also requires a higher muscular activity inducing a higher reaction force. This rapid cyclic movement was chosen because of its simplicity, but is less natural 
than the in vivo recorded arm kinematics. For this latter situation, the resulting reaction force was comparable to in vivo measures recorded by instrumented shoulder prostheses [24,33,34].

The strength of the present work is to confirm the feasibility and potential of the null-space optimization for joint biomechanics problems. The null space allowed that the second optimization do not affect the optimal solution of the fist optimization. Therefore, the first optimization can be solved independently from the second one. This decoupling reduced the complexity of the overall scheme. Because of the simplicity of the musculoskeletal model, the present work has several limitations. The most important is the reduced number of muscles. This limited the range of motion to elevation in the scapular plane. We can indeed assume that this set of muscle is reasonable for this specific movement [2,4,26,35-37]. The stability of the glenohumeral joint is a key issue that was not accounted for in this spherical joint model. We have however verified that the joint reaction force was directed towards the glenoid fossa, represented by an ellipsoid [38] initially inclined by $5^{\circ}$ inferiorly and $5^{\circ}$ posteriorly [39]. In the present model, we minimized the sum of the square muscle force divided by the PCSA. With the quadratic form, the matrix $E$ is the identity and $W^{+}$corresponds to the classical Moore-Penrose pseudo-inverse [40]. We limited our analysis to this objective function, although some authors have proposed other energy-related cost function appeared to lead to more realistic predictions [41], while others have proposed a $\mathrm{min} / \mathrm{max}$ criterion to optimize the muscular fatigue [e.g. 42]. The muscle stress used here is however the most commonly used function [40,43]. Besides, it seems clear that the central nervous system uses different strategies according to the circumstances: optimize energy consumption, fatigue, precision, rapidity, smoothness, etc. In a recent paper, a comparison of computer prediction and EMG measurements confirmed the difficulty to correctly predict the activity of antagonist muscles [44].

The present paper shows that the pseudo-inverse and nullspace operators can be used efficiently to control an over-actuated mechanical system such as the shoulder joint. In a next step, this algorithm will be implemented in an existing shoulder model to predict humeral head translation and joint contact patterns in more complex situation. Such a model should provide key information in the understanding of osteoarthritis occurrence or prosthesis failure, by analyzing the effect of its anatomical and physiological parameters on the articular contact pressure.

\section{Conflict of interest statement}

This study was partly supported by Tornier Inc., Edina, MN.

\section{References}

[1] Inman VT, Saunders JB, Abbott LC. Observations of the function of the shoulder joint. J Bone Joint Surg Am 1944;26:1-30.

[2] Poppen NK, Walker PS. Forces at the glenohumeral joint in abduction. Clin Orthop 1978;135:165-70.

[3] Terrier A, Vogel A, Capezzali M, Farron A. An algorithm to allow humerus translation in the indeterminate problem of shoulder abduction. Med Eng Phys 2008;30(6):710-6.

[4] Terrier A, Reist A, Vogel A, Farron A. Effect of supraspinatus deficiency on humerus translation and glenohumeral contact force during abduction. Clin Biomech 2007;22(6):645-51.

[5] Karlsson D, Peterson B. Towards a model for force predictions in the human shoulder. J Biomech 1992;25(2):189-99.

[6] Fick R. Handbuch des Anatomie und Mechanik der Gelencke. Jena: Gustav Fischer; 1910.

[7] van der Helm FC. Analysis of the kinematic and dynamic behavior of the shoulder mechanism. J Biomech 1994;27(5):527-50.

[8] Charlton IW, Johnson GR. A model for the prediction of the forces at the glenohumeral joint. Proc Inst Mech Eng [H] 2006;220(8):801-12.

[9] Yanagawa T, Goodwin CJ, Shelburne KB, Giphart JE, Torry MR, Pandy MG. Contributions of the individual muscles of the shoulder to glenohumeral joint stability during abduction. J Biomech Eng 2008;130(2):021024.
[10] Holzbaur KR, Murray WM, Delp SL. A model of the upper extremity for simulating musculoskeletal surgery and analyzing neuromuscular control. Ann Biomed Eng 2005;33(6):829-40.

[11] Khatib O, Demircan E, De Sapio V, Sentis L, Besier T, Delp S. Robotics-based synthesis of human motion. J Physiol Paris 2009;103(3-5):211-9.

[12] Dickerson CR, Chaffin DB, Hughes RE. A mathematical musculoskeletal shoulder model for proactive ergonomic analysis. Comput Methods Biomech Biomed Eng 2007;10(6):389-400.

[13] Schouten AC, Mugge W, van der Helm FC. NMClab, a model to assess the contributions of muscle visco-elasticity and afferent feedback to joint dynamics. J Biomech 2008;41(8):1659-67.

[14] Song D, Lan N, Loeb GE, Gordon J. Model-based sensorimotor integration for multi-joint control: development of a virtual arm model. Ann Biomed Eng 2008;36(6):1033-48.

[15] Charlton IW, Johnson GR. Application of spherical and cylindrical wrapping algorithms in a musculoskeletal model of the upper limb. J Biomech 2001;34(9):1209-16.

[16] Marsden SP, Swailes DC, Johnson GR. Algorithms for exact multi-object muscle wrapping and application to the deltoid muscle wrapping around the humerus. Proc Inst Mech Eng [H] 2008;222(7):1081-95.

[17] Liu J, Hughes RE, Smutz WP, Niebur G, Nan-An K. Roles of deltoid and rotator cuff muscles in shoulder elevation. Clin Biomech 1997;12(1):32-8.

[18] Ackland DC, Pak P, Richardson M, Pandy MG. Moment arms of the muscles crossing the anatomical shoulder. J Anat 2008;213(4):383-90.

[19] Favre P, Sheikh R, Fucentese SF, Jacob HA. An algorithm for estimation of shoulder muscle forces for clinical use. Clin Biomech 2005;20(8):822-33.

[20] Veeger HE, Van der Helm FC, Van der Woude LH, Pronk GM, Rozendal RH. Inertia and muscle contraction parameters for musculoskeletal modelling of the shoulder mechanism. J Biomech 1991;24(7):615-29.

[21] Wu G, van der Helm FC, Veeger HE, Makhsous M, Van Roy P, Anglin C, et al. ISB recommendation on definitions of joint coordinate systems of various joints for the reporting of human joint motion. Part II. Shoulder, elbow, wrist and hand. J Biomech 2005;38(5):981-92.

[22] Kawamura S, Kino H, Won C. High-speed manipulation by using parallel wiredriven robots. Robotica 2000;18:13-21.

[23] Crowninshield RD, Brand RA. A physiologically based criterion of muscle force prediction in locomotion. J Biomech 1981;14(11):793-801.

[24] Bergmann G, Graichen F, Bender A, Kaab M, Rohlmann A, Westerhoff P. In vivo glenohumeral contact forces-measurements in the first patient 7 months postoperatively. J Biomech 2007;40(10):2139-49.

[25] Coley B. Shoulder function and outcome evaluation after surgery using 3D inertial sensors. In: School of Engineering. Lausanne: Swiss Federal Institute of Technology; 2007. p. 232.

[26] Terrier A, Reist A, Merlini F, Farron A. Simulated joint and muscle forces in reversed and anatomic shoulder prostheses. J Bone Joint Surg B 2008;90(6):751-6.

[27] Favre P, Jacob HA, Gerber C. Changes in shoulder muscle function with humeral position: a graphical description. J Shoulder Elbow Surg 2009;18(1): 114-21.

[28] Kuechle DK, Newman SR, Itoi E, Niebur GL, Morrey BF, An KN. The relevance of the moment arm of shoulder muscles with respect to axial rotation of the glenohumeral joint in four positions. Clin Biomech (Bristol, Avon) 2000;15(5):322-9.

[29] Kuechle DK, Newman SR, Itoi E, Morrey BF, An KN. Shoulder muscle moment arms during horizontal flexion and elevation. J Shoulder Elbow Surg 1997;6(5):429-39.

[30] Bassett RW, Browne AO, Morrey BF, An KN. Glenohumeral muscle force and moment mechanics in a position of shoulder instability. J Biomech 1990;23(5):405-15.

[31] Graichen H, Englmeier KH, Reiser M, Eckstein F. An in vivo technique for determining 3D muscular moment arms in different joint positions and during muscular activation-application to the supraspinatus. Clin Biomech (Bristol, Avon) 2001;16(5):389-94.

[32] Juul-Kristensen B, Bojsen-Moller F, Finsen L, Eriksson J, Johansson G, Stahlberg $\mathrm{F}$, et al. Muscle sizes and moment arms of rotator cuff muscles determined by magnetic resonance imaging. Cells Tissues Organs 2000;167(2-3):214-22.

[33] Westerhoff P, Graichen F, Bender A, Rohlmann A, Bergmann G. An instrumented implant for in vivo measurement of contact forces and contact moments in the shoulder joint. Med Eng Phys 2009;31(2):207-13.

[34] Westerhoff P, Rohlmann A, Bender A, Graichen F, Bergmann G. In vivo shoulder joint forces at isolated motions. 16th ESB congress. J Biomech 2008. AT-0726.

[35] Terrier A, Merlini F, Pioletti DP, Farron A. Comparison of polyethylene wear in anatomical and reversed shoulder prostheses. J Bone Joint Surg $\mathrm{Br}$ 2009;91:977-82.

[36] Debski RE, McMahon PJ, Thompson WO, Woo SL, Warner JJ, Fu FH. A new dynamic testing apparatus to study glenohumeral joint motion. J Biomech 1995;28(7):869-74.

[37] Kedgley AE, Mackenzie GA, Ferreira LM, Drosdowech DS, King GJ, Faber KJ, et al. The effect of muscle loading on the kinematics of in vitro glenohumeral abduction. J Biomech 2007;40(13):2953-60.

[38] McPherson EJ, Friedman RJ, An YH, Chokesi R, Dooley RL. Anthropometric study of normal glenohumeral relationships. J Shoulder Elbow Surg 1997;6(2):105-12

[39] Rockwood CA, Matsen FA. The shoulder, 2 vols., second ed. Philadelphia: Saunders; 1998,1 CD-ROM

[40] Whitney DE. Resolved motion rate control of manipulators and human prostheses. IEEE Trans Man-Machine Syst 1969;Mm10(2):47-53. 
[41] Praagman M, Chadwick EK, van der Helm FC, Veeger HE. The relationship between two different mechanical cost functions and muscle oxygen consumption. J Biomech 2006;39(4):758-65.

[42] Rasmussen J, Damsgaard M, Voigt $M$. Muscle recruitment by the $\mathrm{min} / \mathrm{max}$ criterion-a comparative numerical study. J Biomech 2001;34(3): 409-15.
[43] Happee R, Van der Helm FC. The control of shoulder muscles during goal directed movements, an inverse dynamic analysis. J Biomech 1995;28(10):1179-91.

[44] Dickerson CR, Hughes RE, Chaffin DB. Experimental evaluation of a computational shoulder musculoskeletal model. Clin Biomech (Bristol, Avon) 2008;23(7):886-94.

Please cite this article in press as: Terrier A, et al. A musculoskeletal shoulder model based on pseudo-inverse and null-space optimization. Med Eng Phys (2010), doi:10.1016/j.medengphy.2010.07.006 